\title{
Correlation of Serum Adipolin with Epicardial Fat Thickness and Severity of Coronary Artery Diseases in Acute Myocardial Infarction and Stable Angina Pectoris Patients
}

\author{
Behzad Babapour $^{\mathrm{a}}$ Hossein Doustkamia ${ }^{\mathrm{a}}$ Leli Avesta ${ }^{\mathrm{a}} \quad$ Alireza Moradi $^{\mathrm{b}}$ \\ Saeideh Saadat ${ }^{c}$ Kiavash Piralaei ${ }^{b}$ Mohammad Reza Aslanid, e \\ a Department of Cardiology, Faculty of Medicine, Ardabil University of Medical Sciences, Ardabil, Iran; \\ ${ }^{b}$ Department of Physiology, Faculty of Medicine, Ardabil University of Medical Sciences, Ardabil, Iran; \\ 'Department of Physiology, School of Medicine, Zahedan University of Medical Sciences, Zahedan, Iran; \\ d Lung Inflammatory Diseases Research Center, Faculty of Medicine, Ardabil University of Medical Sciences,

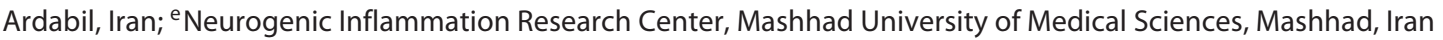

\section{Highlights of the Study}

- Obesity is an important parameter as a risk factor for coronary arteriosclerosis.

- Adipolin (CRTP12) is an adipokine that is highly expressed in adipose and has anti-inflammatory effects.

- Serum adipolin levels have been independently correlated with coronary artery disease.

- We report decreased serum levels of adipolin in acute myocardial infarction cases and a negative correlation between levels of serum adipolin and epicardial fat thickness.

\section{Keywords}

Adipolin - Epicardial fat thickness - Echocardiography · Acute myocardial infarction - Stable angina pectoris .

Angiography

\section{Abstract \\ Objective: Adipolin/C1q/TNF-related protein-12 is a family of CTRPs highly expressed in adipose tissue with glucose- lowering and anti-inflammatory effects. Various risk factors have been suggested in the incidence of cardiovascular dis- eases, such as a decrease in anti-inflammatory or an increase in inflammatory factors. The purpose of the present study was to investigate the correlation of adipolin with anthropo-}

karger@karger.com www.karger.com/mpp

Karger $\stackrel{\text { ' }}{5}$

BOPEN ACCESS
(C) 2020 The Author(s)

Published by S. Karger AG, Basel

This is an Open Access article licensed under the Creative Commons Attribution-NonCommercial-4.0 International License (CC BY-NC) (http://www.karger.com/Services/OpenAccessLicense), applicable to the online version of the article only. Usage and distribution for commercial purposes requires written permission. metric, angiographic, echocardiographic, and biochemical parameters. Subject and Methods: A total of 90 patients who were candidates for angiography were included in the study and divided into 3 groups: 30 patients with acute myocardial infarction (AMI), 30 patients with stable angina pectoris (SAP), and 30 subjects as a control group with a history of chest pain but normal angiography. Anthropometric, angiographic, echocardiographic, and biochemical parameters were measured in all subjects. Results: Serum adipolin levels were significantly decreased in patients with AMI compared with the SAP and control groups ( $p<0.001$ for both). In addition, there was a negative association between serum levels of adipolin and epicardial fat thickness (EFT) and Gensini score in CAD patients. The results of multivariate linear re- 
gression analysis revealed that EFT values were independently associated with serum adipolin levels. Conclusion: The current study showed an independent association of adipolin with EFT for the first time in patients with AMI. Decreased adipolin levels in patients with AMI may be involved in the process of atherosclerosis, which requires further study.

(C) 2020 The Author(s)

Published by S. Karger AG, Basel

\section{Introduction}

Cardiovascular disease is one of the leading causes of death in most countries of the world [1]. Many factors have been implicated in the development of coronary artery disease $(\mathrm{CAD})$, including hypertension, hyperlipidemia, smoking, and family history [2]. Atherosclerosis has also been reported to be the major mechanism involved in CAD [3]. Studies have shown that obesity is an important parameter as a risk factor for coronary arteriosclerosis and consequently for the increased risk of myocardial infarction [4-6]. Different types of proinflammatory or anti-inflammatory mediators, as well as cytokines, are secreted from adipose tissue called adipokines (or adipocytokines) $[7,8]$. These adipokines are involved in various stages of atherosclerosis, such as endothelial dysfunction to plaque destabilization [9].

The C1q/TNF-related protein family (CRTPs) is a set of adipokines that are highly homologous to adiponectin and are largely synthesized in adipose tissue [10]. Adipolin (CRTP12) is an adipokine that is highly expressed in adipose tissue, especially adipocytes. In in vivo studies, adipolin shows anti-inflammatory effects, and in macrophage culture it indicates diminishing effects on the levels of inflammatory cytokines, such as tumor necrosis factor (TNF)- $\alpha$, interleukin (IL)- $1 \beta$, and monocyte chemoattractant protein-1 (MCP-1) [11, 12]. Reportedly, serum adipolin levels can decrease inflammation and obesity status [13]. Very recently, serum adipolin levels have been independently correlated with CAD and may be implicated in the pathogenesis mechanism of atherosclerosis [14].

There are no data on serum adipolin levels in patients with acute myocardial infarction (AMI) and stable angina pectoris (SAP). Bedsides, serum creatine kinase myocardial band (CK-MB) is used clinically to diagnose AMI and also to estimate the extent of myocardial infarction. Due to the lack of an established association between adipolin with CK-MB levels in PCI-treated AMI patients, the purpose of the present study is to evaluate serum adipolin levels in patients with AMI and SAP and their correlation with anthropometric, angiographic, echocardiographic, and biochemical parameters.

\section{Subjects and Methods}

This study was conducted from April 2018 to July 2019 at the Imam Khomeini Educational and Clinical Hospital, Ardabil, Iran. Approval was obtained from the Ethics Committee of Ardabil University of Medical Sciences (IR.ARUMS.REC.1397.197) and written consent was obtained from the patients.

A total of 60 hospitalized patients were enrolled with clinically diagnosed CAD and underwent coronary angiography. Also, 30 patients with chest pain who had normal angiography were included as the control group. All the subjects were male. Patients with a history of hospitalization $\leq 6$ months before the study, history of myocardial infarction, valvular heart diseases, acute or chronic infectious diseases, autoimmune diseases, chronic respiratory diseases, myocarditis, serious heart failure, pericardial effusion, poor echocardiographic imaging, chronic renal failure, hepatitis, cancer, and steroid therapy were excluded from the study.

Patients with a CAD diagnosis were divided into AMI and SAP groups (for both groups $n=30$ ). Inclusion criteria in the AMI group were as follows: significant elevations in troponin- $\mathrm{T}$ and CK-MB, and ST-segment elevation at the J point in at least 2 adjacent leads. On the other hand, the inclusion criteria in the SAP group were: the presence of typical exertion-induced chest discomfort associated with ECG changes during exercise testing with horizontal ST-segment depression of at least $1 \mathrm{~mm}$. The subjects in the control group had previously experienced chest pain, but no changes in the electrocardiographic rhythm or significant coronary stenosis were observed based on coronary angiography examination.

Type 2 diabetes mellitus was defined in the study patients based on its diagnosis or the need for drug therapy. Subjects with systolic blood pressure (SBP) $\geq 140 \mathrm{~mm} \mathrm{Hg}$, diastolic blood pressure (DBP) $\geq 90 \mathrm{~mm} \mathrm{Hg}$, or those treated with antihypertensive drugs were defined as having hypertension. Hyperlipidemia was defined based on the following criteria: high-density lipoprotein cholesterol (HDL-C) $<35 \mathrm{mg} / \mathrm{dL}$, triglycerides $\geq 150 \mathrm{mg} / \mathrm{dL}$, low-density lipoprotein cholesterol (LDL-C) $\geq 100 \mathrm{mg} / \mathrm{dL}$, total cholesterol (TC) $\geq 200 \mathrm{mg} / \mathrm{dL}$, or those undergoing treatment for lipid disorders.

Demographic information, SBP and DBP, height, weight, and abdominal and hip circumference were also measured for all participants. Clinical data were also collected from study participants, including cardiovascular risk factors, medical history, and associated comorbidities. Clinical examinations including body mass index (BMI) and waist-hip ratio (WHR) were also performed for all subjects.

\section{Laboratory Measurements}

In the AMI group, blood samples for biochemistry measurements were collected just after admission. For all groups, blood samples were collected early in the morning after overnight fasting with the subjects in the supine position. Blood samples were collected in tubes containing EDTA. The plasma was immediately centrifuged at $4{ }^{\circ} \mathrm{C}$ and then stored at $-80^{\circ} \mathrm{C}$ until analysis. Plasma 
Table 1. Baseline characteristics and laboratory findings in the study groups

\begin{tabular}{|c|c|c|c|c|}
\hline & $\begin{array}{l}\text { Non-CAD } \\
(n=30)\end{array}$ & $\begin{array}{l}\text { SAP } \\
(n=30)\end{array}$ & $\begin{array}{l}\text { AMI } \\
(n=30)\end{array}$ & $p$ value \\
\hline Age, years & $60.96 \pm 13.35$ & $62.96 \pm 8.52$ & $53.33 \pm 10.23$ & 0.002 \\
\hline BMI & $25.93 \pm 3.06$ & $26.62 \pm 4.46$ & $27.33 \pm 3.41$ & 0.343 \\
\hline Waist circumference, $\mathrm{cm}$ & $93.43 \pm 5.68$ & $96.06 \pm 8.55$ & $97.90 \pm 6.16$ & 0.047 \\
\hline Hip circumference, $\mathrm{cm}$ & $100.56 \pm 4.88$ & $99.73 \pm 8.27$ & $102.03 \pm 8.27$ & 0.471 \\
\hline WHR & $0.92 \pm 0.04$ & $0.96 \pm 0.02$ & $0.96 \pm 0.05$ & 0.004 \\
\hline Systolic BP, mm Hg & $120(115-125)$ & $140(125-150)$ & $120(110-130)$ & 0.000 \\
\hline Diastolic BP, mm Hg & $80(70-80)$ & $80(70-90)$ & $75(61-80)$ & 0.009 \\
\hline Heart rate, bpm & $74(70-79)$ & $74(70-80)$ & $78(74-80)$ & 0.125 \\
\hline $\mathrm{SpO}_{2}, \%$ & $95(95-96)$ & $95(94-96)$ & $96(95-97)$ & 0.006 \\
\hline Smoking & $12(40)$ & $12(40)$ & $15(50)$ & 0.440 \\
\hline Hypertension & $13(43.3)$ & $14(46.7)$ & $8(26.7)$ & 0.190 \\
\hline Diabetes & $3(10)$ & $8(26.7)$ & $6(20)$ & 0.328 \\
\hline Hyperlipedemia & $11(36.7)$ & $19(63.3)$ & $8(26.7)$ & 0.439 \\
\hline Familiar heart disease & $14(46.7)$ & $14(46.7)$ & $13(43.3)$ & 0.798 \\
\hline $\mathrm{TC}, \mathrm{mg} / \mathrm{dL}$ & $153.23 \pm 20.10$ & $167.60 \pm 30.90$ & $172.13 \pm 27.17$ & 0.019 \\
\hline $\mathrm{TG}, \mathrm{mg} / \mathrm{dL}$ & $112.16 \pm 44.14$ & $127.53 \pm 55.28$ & $125.23 \pm 40.47$ & 0.398 \\
\hline HDL-C, mg/dL & $44.53 \pm 7.65$ & $40.40 \pm 7.38$ & $40.06 \pm 5.30$ & 0.393 \\
\hline LDL-C, mg/dL & $80.16 \pm 17.54$ & $98.13 \pm 17.53$ & $105.46 \pm 18.38$ & 0.002 \\
\hline $\mathrm{FBG}, \mathrm{mg} / \mathrm{dL}$ & $97.36 \pm 9.22$ & $101.40 \pm 9.08$ & $99.56 \pm 8.61$ & 0.225 \\
\hline BUN, mg/dL & $36.10 \pm 9.50$ & $41.86 \pm 22.90$ & $43.30 \pm 29.60$ & 0.420 \\
\hline $\mathrm{Cr}, \mathrm{mg} / \mathrm{dL}$ & $1.21 \pm 0.20$ & $1.24 \pm 0.29$ & $1.27 \pm 0.40$ & 0.808 \\
\hline Uric acid, mg/dL & $5.25(4.5-5.9)$ & $5.25(4.4-7)$ & $5.6(5-7)$ & 0.423 \\
\hline Hemoglobin, g/dL & $14.7(13.3-15.2)$ & $14.1(13.5-15.1)$ & $15.1(13.2-16.3)$ & 0.277 \\
\hline $\mathrm{WBC}, 10^{3} / \mathrm{mm}^{3}$ & $7.29 \pm 1.78$ & $8.63 \pm 1.23$ & $9.24 \pm 1.79$ & 0.000 \\
\hline Platelet, $10^{3} / \mathrm{mm}^{3}$ & $195.2 \pm 53.52$ & $223.16 \pm 48.37$ & $312.76 \pm 47.67$ & 0.238 \\
\hline $\mathrm{CK}-\mathrm{MB}, \mathrm{ng} / \mathrm{mL}$ & $2.8(2.5-3.1)$ & $3.5(3-4.6)$ & $36(22-44)$ & 0.000 \\
\hline HsTnT, ng/L & $2.5(1-5)$ & $13(10-14)$ & $32(24-46)$ & 0.000 \\
\hline Gensini score & Not done & $32 \pm 14.76$ & $46.53 \pm 17.01$ & 0.000 \\
\hline Adipolin, pg/mL & $1,185.66 \pm 291.91$ & $929.33 \pm 168.88$ & $715.66 \pm 134.92$ & 0.000 \\
\hline Adipolin (adjusted), pg/mL $\mathrm{mL}^{\mathrm{a}}$ & $1,175.68 \pm 26.71$ & $947.31 \pm 15.75$ & $704.94 \pm 17.86$ & 0.000 \\
\hline \multicolumn{5}{|l|}{ Medication (on admission) } \\
\hline Statin & $10(33.33)$ & $10(33.33)$ & $4(13.33)$ & 0.082 \\
\hline Aspirin & $11(36.7)$ & $15(50)$ & $7(23.33)$ & 0.287 \\
\hline ACEI/ARB & $8(26.66)$ & $12(40)$ & $4(13.33)$ & 0.246 \\
\hline
\end{tabular}

Data are expressed as $n(\%)$, the mean $\pm \mathrm{SD}$, or median (IQR). SAP, stable angina pectoris; AMI, acute myocardial infarction; non-CAD, non-coronary artery diseases; $\mathrm{BMI}$, body mass index; $\mathrm{BP}$, blood pressure; $\mathrm{SpO}_{2}, \mathrm{O}_{2}$ saturation; TC, total cholesterol; TG, triglycerides; LDL-C, low-density lipoprotein cholesterol; HDL-C, highdensity lipoprotein cholesterol; FBG, fasting blood glucose; BUN, blood urea nitrogen; Cr, creatinine; WBC, white blood cell; CK-MB, creatine kinase myocardial band; HsTnT, high-sensitivity troponin-T; ACEI, angiotensin-converting enzyme inhibitor; ARB, angiotensin II receptor blocker.

${ }^{\text {a }}$ Mean \pm SD by a general linear model with adjustment for age, BMI, WHR, and smoking status.

glucose, triglycerides, TC, HDL-C, LDL-C, blood urea nitrogen (BUN), creatinine (Cr), white blood cells (WBC), hemoglobin, platelets, and uric acid were measured using standard commercial methods on a parallel multichannel analyzer. In addition, serum $\mathrm{CK}-\mathrm{MB}$ and troponin-T levels were measured using the standard method. Also, the serum concentration of adipolin was measured using a commercial kit (Crystal Day, Shanghai, China) and an electrochemiluminescence method with an Elecsys 2010 Automated Analyzer (Roche Diagnostics).

\section{Echocardiography}

All echocardiographic assessments of study subjects were performed by a cardiologist who was blinded to the clinical information of the patients. Before performing angiography, echocardiography was accompanied using a Philips device manufactured in the USA. All echocardiographic tests were recorded and reviewed by 2 other cardiologists who were blinded to the clinical information of the patients. All measured echocardiographic parameters (left ventricular ejection fraction [LVEF], tricuspid annular plane sys- 


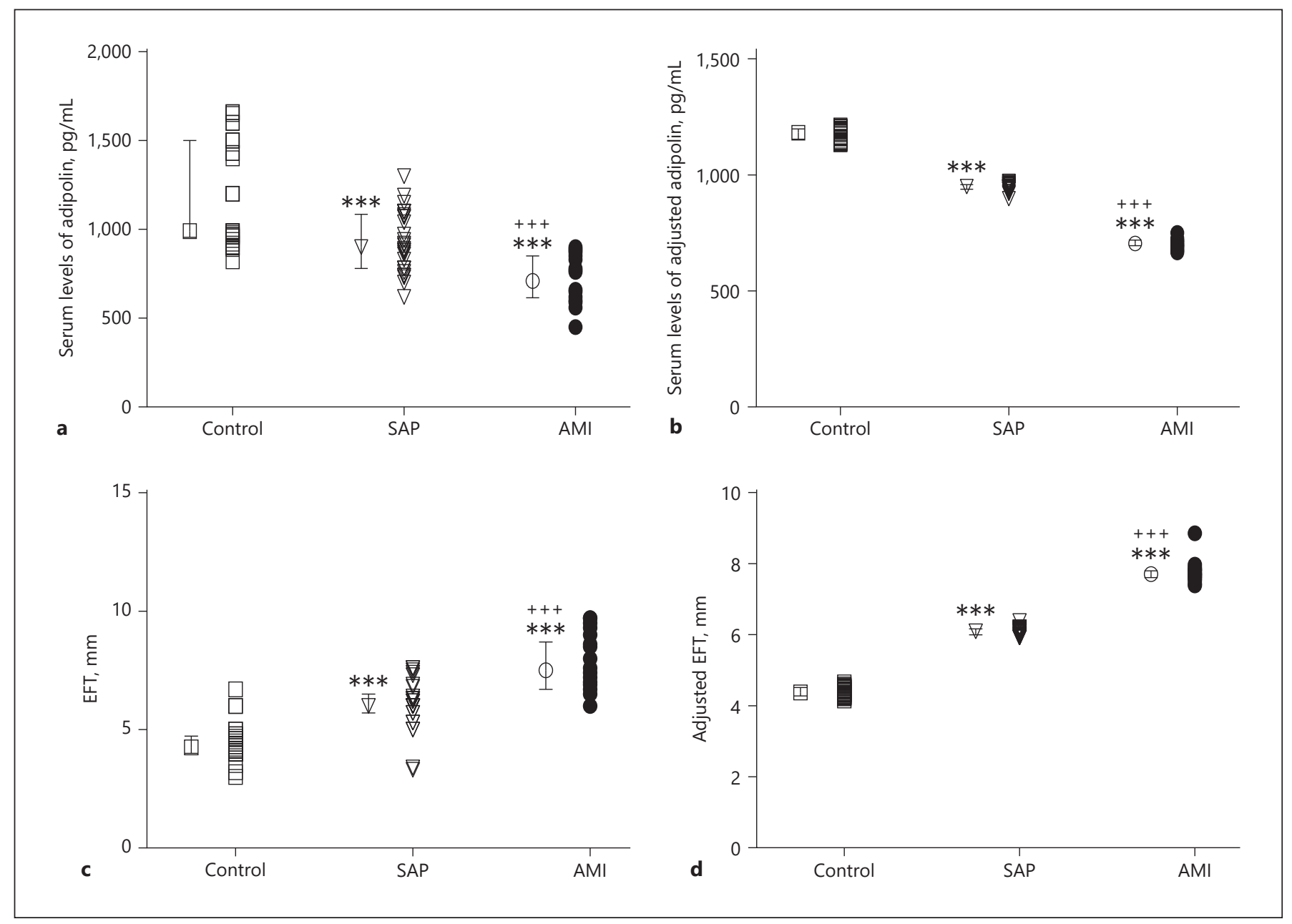

Fig. 1. Individual values and mean \pm SD of serum levels of: adipolin (a), adjusted adipolin (b), baseline EFT (c), and adjusted EFT (d). The adjusting was performed for age, BMI, WHR, and smoking history in the study groups. ${ }^{* * *} p<0.001$, significant statistical difference between the control group and other groups; ${ }^{+++} p<0.001$, significant statistical difference between SAP and AMI.

tolic excursion, tricuspid lateral annular systolic velocity, mitral valve septal annular systolic velocity [e' septal], and mitral valve lateral annular systolic velocity [ $\mathrm{e}^{\prime}$ lateral]) were performed according to the recommendations of the American Society of Echocardiography [15].

The epicardial fat thickness (EFT) is the echo-free space between the outer surface of the right ventricular free wall and visceral pericardium. EFT was measured in at least 3 consecutive beats parallel to the aortic valve and perpendicular to the right ventricular free wall at the side with the greatest thickness on $2 \mathrm{D}$ images [16]. Images were stored in an echo machine then evaluated by an echocardiologist and 2 other cardiologists who were blinded to the clinical status and angiographic results of the selected patients.

\section{Coronary Angiography}

Selective left and right coronary angiography were performed via the radial or femoral artery using the standard Judkins tech- nique with 5- or 6-Fr catheters (Medtronic, Santa Ana, CA, USA) and an Axiom Artis dFA system (Siemens Corp., Berlin, Germany). The modified Gensini score was used to determine the severity of CAD, which is based on the location and degree of stenosis and used in patients with CAD [17].

\section{Statistical Analysis}

The sample size was calculated based on the averages comparison formula with $\alpha=0.05$ and $\beta=0.1, \mu 1=17.62, S 1=38.2, \mu 2=$ 11.9 , and $\mathrm{S} 2=32.03$ from a previous study for the serum levels of the adipolin variable. A sample size of 29 subjects in each group was calculated. The results are given as the mean $\pm \mathrm{SD}$, or median and the 25th to 75th percentiles. Continuous variables were compared using the Student $t$ test. Comparison between groups was made by the Kruskal-Wallis test. If the difference was statistically significant, it was followed by the Mann-Whitney U test for post hoc analysis; alternatively, the ANOVA test was performed with the Tukey-Kramer post hoc test. General linear modeling function 
analysis was used to adjust for age, BMI, WHR, and smoking status. Correlation coefficients were assessed using the Pearson's (or Spearman's rank order) correlation test. Linear regression analyses were performed using adipolin as the dependent variable and biochemical and clinical findings as independent variables. A value of $p<0.05$ was considered to be statistically significant. SPSS version 16.0 and GraphPad Prism 7 software were used for the statistical analysis.

\section{Results}

\section{Patient Characteristics}

The clinical and demographic characteristics of the 3 groups are presented in Table 1 . The mean age of the AMI group was significantly lower than that of the SAP and control groups. The results showed that in patients with AMI and SAP there was a significant increase in levels of troponin-T, CK-MB, LDL-C, and cholesterol, as well as WBC count and WHR compared to the non-CAD group. It was also found that SBP and DBP values were significantly higher in the SAP group compared to the AMI and non-CAD groups. In addition, the amount of $\mathrm{SpO}_{2}$ in the non-CAD and AMI groups was significantly higher than in the SAP group. Interestingly, serum levels of CK-MB and troponin-T were higher in the AMI group than in the SAP group. AMI patients, SAP patients, and non-CAD subjects did not differ significantly with respect to BMI, heart rate, smoking status, diabetes mellitus, hypertension, hyperlipidemia, familiar heart disease history, triglycerides, HDL-C, fasting blood glucose (FBG), BUN, $\mathrm{Cr}$, uric acid, hemoglobin, and platelet count (Table 1).

The serum adipolin levels were significantly lower in the AMI group than in the SAP and non-CAD groups $(p<0.001$ for both; Fig. 1a). Furthermore, there was a significant difference in adipolin serum level between SAP and non-CAD subjects ( $p<0.001$; Fig. 1a). In addition, the serum adipolin levels remained significantly different after adjustment for age, BMI, smoking history, and WHR for the study groups ( $p<0.001$; Fig. $1 b)$.

The echocardiography results are summarized in Table 2. In the AMI and SAP groups, LVEF values were significantly lower than in the non-CAD group. Also, echocardiographic examinations in relation to EFT showed that in the AMI group it was higher and statistically significant compared to the SAP and non-CAD groups ( $p<$ 0.001 for both; Fig. 1c). It was also found that the EFT value in the SAP group was significantly higher than the control group ( $p<0.001$; Fig. 1c). The EFT value remained significantly different after adjustment for age, BMI, smoking history, and WHR for the study groups $(p<0.001$; Fig. 1d).

Table 2. Echocardiography summary of the study groups

\begin{tabular}{lcccc}
\hline & Non-CAD & SAP & AMI & $p$ value \\
\hline LVEF, \% & $55(45-60)$ & $42.5(35-50)$ & $35(30-40)$ & 0.000 \\
$\mathrm{e}^{\prime}$ septal, cm/s & $6.4(5.6-7.5)$ & $6.15(4.8-7)$ & $7(5.2-8.2)$ & 0.247 \\
$\mathrm{e}^{\prime}$ lateral, cm/s & $9.9(8.2-11)$ & $9.4(8.6-11.6)$ & $8.95(8-10)$ & 0.580 \\
TAPSE, mm & $19.49 \pm 3.39$ & $17.72 \pm 3.11$ & $17.84 \pm 3.23$ & 0.068 \\
TV TDI, cm/s & $11.56 \pm 1.57$ & $12.10 \pm 1.75$ & $11.19 \pm 2.12$ & 0.162 \\
EFT, mm & $4.40 \pm 0.77$ & $6.05 \pm 1.01$ & $7.72 \pm 1.22$ & 0.000 \\
EFT (adjusted), mm ${ }^{\text {a }}$ & $4.39 \pm 0.13$ & $6.08 \pm 0.10$ & $7.72 \pm 0.25$ & 0.000 \\
EFT index, mm $/ \mathrm{m}^{2}$ & $0.76 \pm 0.15$ & $1.01 \pm 0.21$ & $1.25 \pm 0.21$ & 0.000
\end{tabular}

Data are expressed as the mean \pm SD or median (IQR). LVEF, left ventricular ejection fraction; $e^{\prime}$ septal, mitral valve septal annular systolic velocity; $\mathrm{e}^{\prime}$ lateral, mitral valve lateral annular systolic velocity; TAPSE, tricuspid annular plane systolic excursion; TV TDI, tricuspid lateral annular systolic velocity; EFT, epicardial fat thickness.

${ }^{a}$ Mean \pm SD by general linear model with adjustment for age, BMI, WHR, and smoking status.

Fig. 2. Pearson's correlation analysis (or Spearman's rank order) of adipolin and: WHR value (correlation coefficient $=-0.275, p=0.009 ; \mathbf{a}$ ), FBG serum levels (correlation coefficient $=-0.517, p=0.000 ; \mathbf{b}$ ), cholesterol serum levels (correlation coefficient $=-0.222, p=0.035 ; \mathbf{c})$, LDL-C serum levels (correlation coefficient $=-0.447, p=0.000 ; \mathbf{d}$ ), $\mathrm{CK}-\mathrm{MB}$ (correlation coefficient $=-0.583, p=0.000 ; \mathbf{e})$, troponin- $\mathrm{T}$ (correlation coefficient $=-0.648, p=0.000 ; \mathbf{f}$ ), WBC count (correlation coefficient $=-0.588, p=0.000 ; \mathbf{g}$ ), Gensini score (correlation coefficient $=-0.592, p=0.000$; h), LVEF (correlation coefficient $=0.562, p=0.000 ; \mathbf{i}$ ), and EFT value (correlation coefficient $=-0.751, p=0.000 ; \mathbf{j}$ ).

(For figure see next page.) 


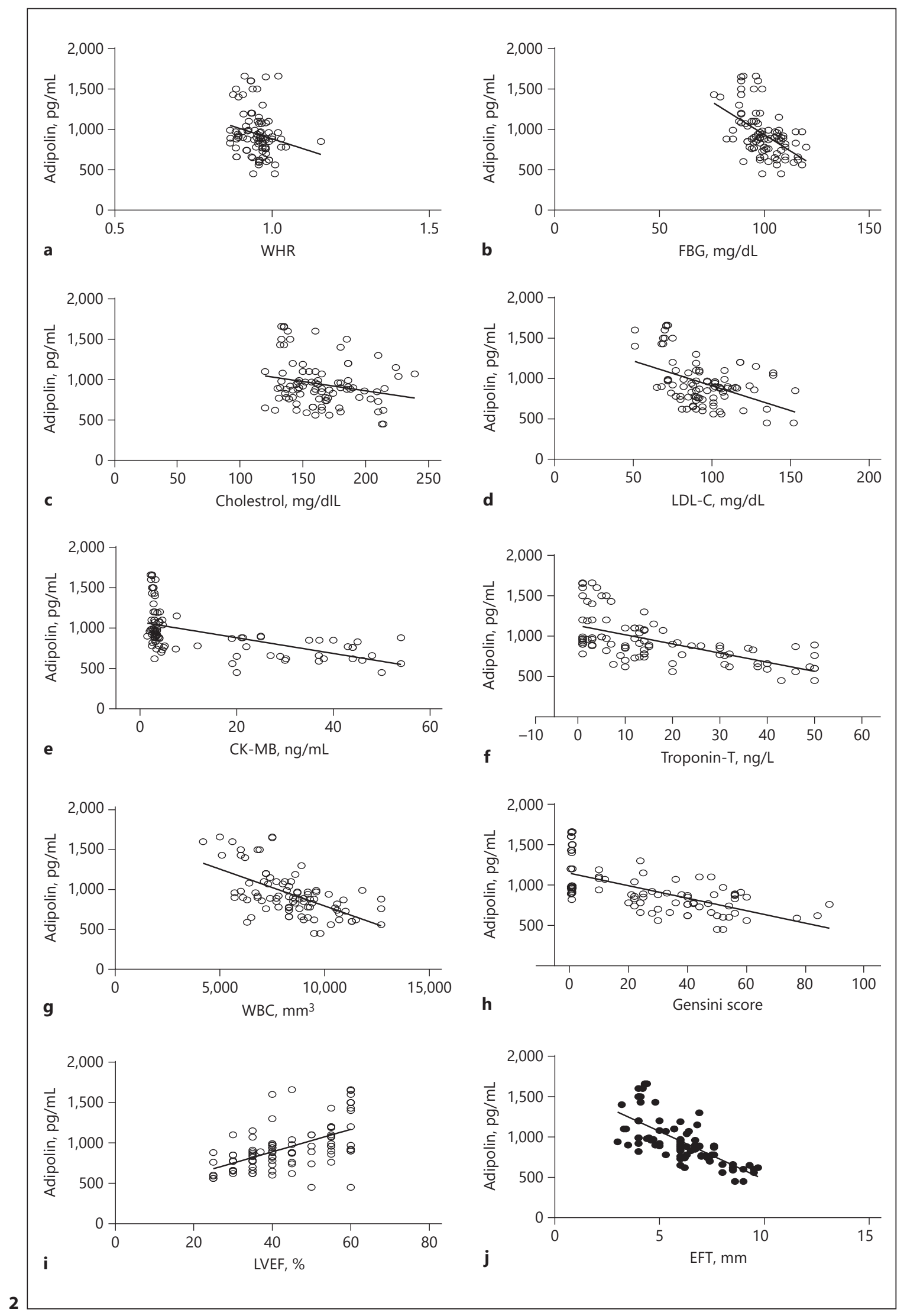


Table 3. Correlation coefficients between serum adipolin and relevant parameters in the study subjects

\begin{tabular}{|c|c|c|}
\hline & \multicolumn{2}{|c|}{ Adipolin } \\
\hline & $r$ & $p$ value \\
\hline Age (years) & 0.195 & 0.066 \\
\hline BMI & -0.111 & 0.299 \\
\hline WHR & -0.275 & 0.009 \\
\hline SBP & 0.095 & 0.373 \\
\hline DBP & 0.202 & 0.057 \\
\hline Heart rate & -0.190 & 0.073 \\
\hline $\mathrm{SpO}_{2}$ & 0.023 & 0.830 \\
\hline FBG & -0.517 & 0.000 \\
\hline $\mathrm{TC}$ & -0.222 & 0.035 \\
\hline TG & -0.053 & 0.617 \\
\hline HDL-C & 0.0 .32 & 0.766 \\
\hline LDL-C & -0.447 & 0.000 \\
\hline CK-MB (ng/mL) ${ }^{\mathrm{a}}$ & -0.583 & 0.000 \\
\hline Troponin-T $(\mathrm{ng} / \mathrm{mL})^{\mathrm{a}}$ & -0.648 & 0.000 \\
\hline WBC $\left(10^{3} / \mathrm{mm}^{3}\right)$ & -0.588 & 0.000 \\
\hline Hemoglobin (g/dL) & -0.082 & 0.441 \\
\hline Platelets $\left(10^{3} / \mathrm{mm}^{3}\right)$ & -0.168 & 0.114 \\
\hline Gensini score & -0.592 & 0.000 \\
\hline $\operatorname{LVEF}(\%)^{\mathrm{a}}$ & 0.562 & 0.000 \\
\hline $\mathbf{e}^{\prime}$ septal $(\mathrm{cm} / \mathrm{s})$ & 0.066 & 0.534 \\
\hline $\mathbf{e}^{\prime}$ lateral $(\mathrm{cm} / \mathrm{s})$ & 0.091 & 0.392 \\
\hline TAPSE (mm) & 0.070 & 0.512 \\
\hline $\operatorname{TV} \operatorname{TDI}(\mathrm{cm} / \mathrm{s})$ & 0.070 & 0.515 \\
\hline $\operatorname{EFT}(\mathrm{mm})$ & -0.751 & 0.000 \\
\hline
\end{tabular}

BMI, body mass index; WHR, waist-hip ratio; SBP, systolic blood pressure; $\mathrm{DBP}$, diastolic blood pressure; $\mathrm{SpO}_{2}, \mathrm{O}_{2}$ saturation; FBG, fasting blood glucose; TC, total cholesterol; TG, triglycerides; LDL-C, low-density lipoprotein cholesterol; HDL-C, high-density lipoprotein cholesterol; CK-MB, creatine kinase myocardial band; WBC, white blood cell; LVEF, left ventricular ejection fraction; $\mathrm{e}^{\prime}$ septal, mitral valve septal annular systolic velocity; $e^{\prime}$ lateral, mitral valve lateral annular systolic velocity; TAPSE, tricuspid annular plane systolic excursion; TV TDI, tricuspid lateral annular systolic velocity; EFT, epicardial fat thickness.

${ }^{a}$ Logarithmic transformation was performed.

\section{Association of Adipolin with Other Factors}

Pearson's (or Spearman's rank) correlation analysis revealed that the level of adipolin is significantly associated with WHR $(r=-0.275, p<0.01$; Fig. $2 \mathrm{a})$, FBG $(r=$ $-0.517, p<0.001$; Fig. $2 b)$, TC $(r=-0.222, p<0.05$; Fig. 2c), LDL-C ( $r=-0.447, p<0.001$; Fig. $2 d)$, CK-MB $(r=-0.583, p<0.001$; Fig. 2e), troponin- $\mathrm{T}(r=-0.648$, $p<0.001$; Fig. 2f), WBC count $(r=-0.648, p<0.001$; Fig. 2g), Gensini score ( $r=-0.592, p<0.001$; Fig. 2h), $\operatorname{LVEF}(r=0.562, p<0.001$; Fig. $2 \mathrm{i})$, and EFT $(r=-0.751$, $p<0.001$; Fig. $2 \mathrm{j}$ ). However, no statistically significant correlation was observed between the levels of serum ad-
Table 4. Multivariable regression analysis of serum adipolin with other factors

\begin{tabular}{llll}
\hline \multicolumn{4}{l}{ Multivariate analysis } \\
\cline { 2 - 4 } & $\beta$ & $95 \%$ CI & $p$ value \\
\hline FBG & -0.326 & -14.37 to -6.10 & 0.000 \\
LDL-C & -0.219 & -5.36 to -0.66 & 0.013 \\
WBC & -0.212 & -0.05 to -0.01 & 0.004 \\
EFT & -0.313 & -91.94 to -12.56 & 0.011 \\
CK-MB $(\mathrm{ng} / \mathrm{mL})^{\mathrm{a}}$ & -0.198 & -234 to 4.87 & 0.060 \\
Troponin-T $(\mathrm{ng} / \mathrm{mL})^{\mathrm{a}}$ & 0.014 & -105 to 119 & 0.903 \\
Gensini score & -0.009 & -2.58 to 2.81 & 0.933 \\
LVEF & 0.063 & -3.10 to 6.31 & 0.501 \\
TC & 0.046 & -1.12 to 2.07 & 0.556 \\
WHR & 0.064 & -411 to 1207 & 0.331 \\
\hline
\end{tabular}

${ }^{\text {a }}$ Logarithmic transformation was performed.

ipolin and age, BMI, SBP, DBP, heart rate, $\mathrm{SpO}_{2}$, plasma glucose, lipid profile, hemoglobin, and platelets (Table 3 ).

In multivariable linear regression analysis, levels of FBG ( $\beta-0.326 ; 95 \%$ CIs -14.37 to $-6.10 ; p=0.000)$, WBC count $(\beta-0.212$; $95 \%$ CIs -0.05 to $-0.01 ; p=0.004)$, LDL$\mathrm{C}(\beta-0.219 ; 95 \%$ CIs -5.36 to $-0.66 ; p=0.013)$, and EFT $(\beta-0.313$; 95\% CIs -91.94 to -12.56 ; $p=0.011)$ were independently associated with adipolin (Table 4). We did not find any significant association between serum adipolin levels and other factors.

\section{Discussion}

In this study: (i) serum adipolin levels were significantly decreased in patients with AMI and SAP, markedly so in AMI patients; (ii) there was a negative association between the serum level of adipolin and WHR, WBC count, CK-MB, troponin-T, FBG, LDL-C, TC, EFT, and Gensini score, but a positive association between serum adipolin level and LVEF, and (iii) the results of multivariate linear regression analysis revealed that FBG, LDL-C, WBC count, and EFT values were independently associated with serum adipolin levels. Indeed, the results indicated that there was a relationship between serum adipolin levels and the severity of CAD based on the Gensini score as well as with EFT in patients with AMI and SAP, markedly so in AMI patients.

Adipolin (CTRP12) is a CTRP protein that is mainly produced by adipocytes. Human and animal studies have reported reduced levels of gene expression and serum adipolin levels in obesity and type 2 diabetes, espe- 
cially in hyperglycemia and increased waist circumference conditions [11]. The results of the present study showed that serum adipolin levels decreased in patients with AMI and SAP compared to non-CAD subjects. Since there was an association between serum adipolin levels with covariates such as FBG, BMI, and WHR, we adjusted them for serum adipolin levels. It was found that the decrease in serum levels of adipolin was independent of the FBG and other covariates in the study population.

Fadaei et al. [14] recently investigated the association between adipolin and CAD compared to that of nonCAD subjects. They showed that patients with CAD have decreased levels of adipolin, which is associated with levels of insulin resistance, adiponectin, inflammatory cytokines, and BMI. The results of our study are consistent with their study regarding a decrease in adipolin levels in patients with AMI and SAP, which was particularly significant in patients with AMI. Although the precise mechanism of this association between adipolin and CAD is unclear, it is likely that factors involved in the relationship between adipokines and atherosclerosis are endothelial dysfunction, inflammation, and lipid metabolism [18].

There are no reports on the association of serum adipolin levels with EFT, as well as with echocardiographic and angiographic findings in AMI and SAP patients. Here we have shown a negative association between adipolin levels and EFT. There was also a positive association between adipolin and LVEF. Serum adipolin levels were lower in patients with AMI than in patients with SAP and non-CAD. There was a significant correlation between adipolin and EFT. Bedsides, multivariate regression analysis presented EFT as an independent variable for adipolin levels.

Obesity has been shown to play a key role in many chronic inflammatory diseases, such as asthma, COPD, rheumatoid arthritis, and CAD [19-21]. EFT echocardiography is an independent measure of visceral fat that has been identified in previous studies to be higher in subjects with higher WHR [22]. Several studies have shown that EFT is an important source of proinflammatory, anti-inflammatory, proatherogenic, and adipokine factors, including IL-6, TNF- $\alpha$, MCP-1, leptin, omentin, resistin, visfatin, angiotensinogen, adiponectin, plasminogen activator inhibitor- 1 , and nerve growth factor $[23,24]$. EFT can affect arthrosclerosis through paracrine or endocrine effects. In addition, patients with CAD have shown a higher EFT compared to non-CAD subjects. Interestingly, according to the Gensini score, EFT has been found to be associated with the severity of CAD [22].

Serum Adipolin in Acute Myocardial Infarction
To the best of our knowledge, this is the first study to show that adipolin levels are associated with levels of troponin-T and CK-MB. According to the results of the present study, adipolin levels decreased with increasing Gensini score in AMI and SAP patients, and markedly so in AMI patients. Although there has been no report on the decreased serum adipolin levels in patients with AMI, the association of the CTRP family with CAD, especially adiponectin, has been reported in several studies [25]. The anti-atherosclerotic effects of adiponectin have been shown in many studies through various mechanisms, such as inhibition of adhesion of monocytes to the endothelial cells, foam cell formation of macrophages, and proliferation of vascular smooth muscle cells $[26,27]$. Alipoor et al. [28] also demonstrated the protective effects of adipolin on vascular remodeling by reducing the macrophage inflammatory response. Since this is the first study to indicate a link between CK-MB and CAD severity based on the Gensini score with serum adipolin levels, the precise mechanism of this association is unclear and needs further study.

Serum adipolin levels in the present study were similar to those in some studies [14] and different from others [28]. The reason for this difference may be due to the influence of various factors, such as demographic differences, different conditions, underlying diseases, kit calibration, and other underlying disease-related factors. Therefore, in research studies, it seems that each laboratory needs a control group to report its normal values to determine the normal range.

The current study has several limitations. First, we did not include women in the study and thus did not determine the gender effect on serum adipolin levels and their association with the severity of the disease. Previous studies have shown that adipolin serum levels are higher in women than in men [14]. Second, in vivo and in vitro studies have shown that adipolin can have a protective effect against proinflammatory cytokines. It is advisable to test the direct effects of adipolin on the proinflammatory cytokines in underlying CAD conditions. Finally, the sample size of our study was modest and thus it will be necessary to perform evaluations with a larger sample size.

\section{Conclusion}

The current study revealed an independent association of adipolin with EFT for the first time in AMI and SAP patients. In addition, these results represent a rela-

Med Princ Pract 2021;30:52-61

DOI: $10.1159 / 000508834$ 
tionship between adipolin and WHR, WBC count, CK$\mathrm{MB}$, troponin-T, FBG, LDL-C, TC, EFT, Gensini score, and LVEF. Various factors, such as atherosclerosis, are involved in the pathogenesis of CAD. It has been suggested that a decrease in adipolin through inflammation and alteration in the lipoprotein metabolism may influence the process of atherosclerosis. Although this study has not shown a causal link between adipolin and CAD in patients with AMI, it suggests a role for adipolin in these patients.

\section{Acknowledgments}

This work was financially supported by the Ardabil University of Medical Sciences. This report is based on a study entitled "Evaluation of Serum Adipolin Levels with Echocardiography and Angiography Findings in Acute Myocardial Infarction Patients and Comparison of Their Results with Stable Angina Patients," registered by the Research Committee of Ardabil University of Medical Sciences.

\section{Statement of Ethics}

This study was conducted after gaining approval from the Ethics Committee of Ardabil University of Medical Sciences (IR.ARUMS.REC.1397.197).

\section{Disclosure Statement}

The authors have no conflicts of interest to declare.

\section{Author Contributions}

M.R.A., B.B., H.D., and L.A.: literature search, proposal writing, data collection, analysis of data, interpretation of data, manuscript preparation, review of the manuscript. A.M. and K.P.: data collection, analysis, draft preparation. S.S.: analysis, draft preparation, review of the manuscript.

\section{References}

1 Sanchis-Gomar F, Perez-Quilis C, Leischik R, Lucia A. Epidemiology of coronary heart disease and acute coronary syndrome. Ann Transl Med. 2016 Jul;4(13):256.

2 Veeranna V, Pradhan J, Niraj A, Fakhry H, Afonso L. Traditional cardiovascular risk factors and severity of angiographic coronary artery disease in the elderly. Prev Cardiol. 2010; 13(3):135-40.

3 Al-Zakwani I, Al Siyabi E, Alrawahi N, AlMulla A, Alnaeemi A, Shehab A, et al. Association between peripheral artery disease and major adverse cardiovascular events in patients with acute coronary syndrome: findings from the Gulf COAST Registry. Med Princ Pract. 2019;28(5):410-7.

4 Wang Q, Rao S, Shen GQ, Li L, Moliterno DJ, Newby LK, et al. Premature myocardial infarction novel susceptibility locus on chromosome 1P34-36 identified by genomewide linkage analysis. Am J Hum Genet. 2004 Feb; 74(2):262-71.

5 Liu SW, Qiao SB, Yuan JS, Liu DQ. Association of plasma visfatin levels with inflammation, atherosclerosis and acute coronary syndromes (ACS) in humans. Clin Endocrinol (Oxf). 2009 Aug;71(2):202-7.

6 Gulgun M. Aortic stiffness may be affected by body mass index. Med Princ Pract. 2017; 26(5):495-95.

7 Aslani MR, Keyhanmanesh R, Alipour MR. Increased visfatin expression is associated with nuclear factor- $\kappa \mathrm{B}$ in obese ovalbuminsensitized male Wistar Rat Tracheae. Med Princ Pract. 2017;26(4):351-8.
8 Aslani MR, Ghobadi H, Panahpour H, Ahmadi M, Khaksar M, Heidarzadeh M. Modification of lung endoplasmic reticulum genes expression and NF-kB protein levels in obese ovalbumin-sensitized male and female rats. Life Sci. 2020 Apr;247:117446.

9 Van de Voorde J, Pauwels B, Boydens C, Decaluwé K. Adipocytokines in relation to cardiovascular disease. Metabolism. 2013 Nov; 62(11):1513-21.

10 Wong GW, Wang J, Hug C, Tsao TS, Lodish HF. A family of Acrp30/adiponectin structural and functional paralogs. Proc Natl Acad Sci USA. 2004 Jul;101(28):10302-7.

11 Enomoto T, Ohashi K, Shibata R, Higuchi A, Maruyama S, Izumiya Y, et al. Adipolin/ C1qdc2/CTRP12 protein functions as an adipokine that improves glucose metabolism. J Biol Chem. 2011 Oct;286(40):34552-8.

12 Wei Z, Peterson JM, Lei X, Cebotaru L, Wolfgang MJ, Baldeviano GC, et al. C1q/TNF-related protein-12 (CTRP12), a novel adipokine that improves insulin sensitivity and glycemic control in mouse models of obesity and diabetes. J Biol Chem. 2012 Mar;287(13):10301-15.

13 Ohashi K, Shibata R, Murohara T, Ouchi N. Role of anti-inflammatory adipokines in obesity-related diseases. Trends Endocrinol Metab. 2014 Jul;25(7):348-55.

14 Fadaei R, Moradi N, Kazemi T, Chamani E, Azdaki N, Moezibady SA, et al. Decreased serum levels of CTRP12/adipolin in patients with coronary artery disease in relation to inflammatory cytokines and insulin resistance. Cytokine. 2019 Jan;113:326-31.
15 Nagueh SF, Smiseth OA, Appleton CP, Byrd BF 3rd, Dokainish H, Edvardsen T, et al. Recommendations for the evaluation of left ventricular diastolic function by echocardiography: an update from the american society of echocardiography and the european association of cardiovascular imaging. J Am Soc Echocardiogr. 2016 Apr;29(4):277-314.

16 Iacobellis G, Assael F, Ribaudo MC, Zappaterreno A, Alessi G, Di Mario U, et al. Epicardial fat from echocardiography: a new method for visceral adipose tissue prediction. Obes Res. 2003 Feb;11(2):304-10.

17 Sullivan DR, Marwick TH, Freedman SB. A new method of scoring coronary angiograms to reflect extent of coronary atherosclerosis and improve correlation with major risk factors. Am Heart J. 1990 Jun;119(6):1262-7.

18 Seldin MM, Tan SY, Wong GW. Metabolic function of the CTRP family of hormones. Rev Endocr Metab Disord. 2014 Jun;15(2):111-23.

19 Aslani MR, Keyhanmanesh R, Khamaneh AM, Abbasi MM, Fallahi M, Alipour MR. Tracheal overexpression of IL-1 $\beta$, IRAK-1 and TRAF-6 mRNA in obese-asthmatic male Wistar rats. Iran J Basic Med Sci. 2016 Apr;19(4):350-7.

20 Akhavanakbari G, Babapour B, Alipour MR, Keyhanmanesh R, Ahmadi M, Aslani MR. Effect of high fat diet on NF-кB microRNA146a negative feedback loop in ovalbumin-sensitized rats. Biofactors. 2019 Jan;45(1):75-84.

21 Aslani MR, Ghazaei Z, Ghobadi H. Correlation of serum fatty acid binding protein- 4 and interleukin-6 with airflow limitation and quality of life in stable and acute exacerbation of COPD. Turk J Med Sci. 2020 Apr;50(2):337-45.
60

Med Princ Pract 2021;30:52-61

DOI: $10.1159 / 000508834$
Babapour/Doustkami/Avesta/Moradi/ Saadat/Piralaei/Aslani 
22 Iacobellis G, Willens HJ. Echocardiographic epicardial fat: a review of research and clinical applications. J Am Soc Echocardiogr. 2009 Dec;22(12):1311-9.

23 Mazurek T, Zhang L, Zalewski A, Mannion JD, Diehl JT, Arafat H, et al. Human epicardial adipose tissue is a source of inflammatory mediators. Circulation. 2003 Nov; 108(20): 2460-6.

24 Baker AR, Silva NF, Quinn DW, Harte AL, Pagano D, Bonser RS, et al. Human epicardial adipose tissue expresses a pathogenic profile of adipocytokines in patients with cardiovascular disease. Cardiovasc Diabetol. 2006 Jan; 5(1):1.
25 Natsukawa T, Maeda N, Fukuda S, Yamaoka M, Fujishima Y, Nagao H, et al. Significant association of serum adiponectin and creatine kinase-mb levels in st-segment elevation myocardial infarction. J Atheroscler Thromb. 2017 Aug;24(8):793-803.

26 Ouchi N, Kihara S, Arita Y, Nishida M, Matsuyama A, Okamoto Y, et al. Adipocyte-derived plasma protein, adiponectin, suppresses lipid accumulation and class A scavenger receptor expression in human monocyte-derived macrophages. Circulation. 2001 Feb; 103(8):1057-63.
27 Arita Y, Kihara S, Ouchi N, Maeda K, Kuriyama $\mathrm{H}$, Okamoto $\mathrm{Y}$, et al. Adipocyte-derived plasma protein adiponectin acts as a plateletderived growth factor-BB-binding protein and regulates growth factor-induced common postreceptor signal in vascular smooth muscle cell. Circulation. 2002 Jun;105(24): 2893-8.

28 Alipoor E, Salmani M, Yaseri M, KolahdouzMohammadi R, Esteghamati A, Hosseinzadeh-Attar MJ. Role of type 2 diabetes and hemodialysis in serum adipolin concentrations: a preliminary study. Hemodial Int. 2019 Oct; 23(4):472-8. 